\title{
Sorption, Structure and Dynamics of $\mathrm{CO}_{2}$ and Ethane in Silicalite at High Pressure: A Combined Monte Carlo and Molecular Dynamics Simulation Study
}

\author{
Siddharth Gautam *(D), Tingting Liu and David Cole \\ School of Earth Sciences, The Ohio State University, Columbus, OH 43210, USA; liu.2189@osu.edu (T.L.); \\ cole.618@osu.edu (D.C.) \\ * Correspondence: gautam.25@osu.edu; Tel.: +1-614-292-7365
}

Received: 30 November 2018; Accepted: 25 December 2018; Published: 28 December 2018

\begin{abstract}
Silicalite is an important nanoporous material that finds applications in several industries, including gas separation and catalysis. While the sorption, structure, and dynamics of several molecules confined in the pores of silicalite have been reported, most of these studies have been restricted to low pressures. Here we report a comparative study of sorption, structure, and dynamics of $\mathrm{CO}_{2}$ and ethane in silicalite at high pressures (up to 100 bar) using a combination of Monte Carlo (MC) and molecular dynamics (MD) simulations. The behavior of the two fluids is studied in terms of the simulated sorption isotherms, the positional and orientational distribution of sorbed molecules in silicalite, and their translational diffusion, vibrational spectra, and rotational motion. Both $\mathrm{CO}_{2}$ and ethane are found to exhibit orientational ordering in silicalite pores; however, at high pressures, while $\mathrm{CO}_{2}$ prefers to reside in the channel intersections, ethane molecules reside mostly in the sinusoidal channels. While $\mathrm{CO}_{2}$ exhibits a higher self-diffusion coefficient than ethane at low pressures, at high pressures, it becomes slower than ethane. Both $\mathrm{CO}_{2}$ and ethane exhibit rotational motion at two time scales. At both time scales, the rotational motion of ethane is faster. The differences observed here in the behavior of $\mathrm{CO}_{2}$ and ethane in silicalite pores can be seen as a consequence of an interplay of the kinetic diameter of the two molecules and the quadrupole moment of $\mathrm{CO}_{2}$.
\end{abstract}

Keywords: sorption; molecular dynamics; Monte Carlo; $\mathrm{CO}_{2}$; ethane; silicalite

\section{Introduction}

The behavior of molecules is strongly determined by their environment, and is different if they are surrounded by molecules of the same species or of a different species. The latter scenario occurs, for example, when a fluid is confined in a porous media, or is adsorbed on the surface of a substrate. The specific interaction between the fluid molecules and the confining substrate alters the behavior of the fluid. As most of the interesting chemical reactions occur at interfaces between two species, the behavior of molecules under confinement or at an interface in general is an interesting topic of research [1-5]. An important component that determines the interaction between molecules in general is the distribution of the electrostatic charge. In some molecules such as water, an asymmetrical distribution of charge gives rise to a permanent dipole moment. The distribution of charge in a molecule plays an important role in determining the dynamical behavior. For example, it has been found that for molecules of similar shapes and sizes, both the translational and the rotational motions are severely affected by an asymmetry in the charge distribution [6,7]. The motion of acetonitrile, acetaldehyde, and acetone, all with a non-zero dipole moment was found to be severely restricted, whereas that of propane with no dipole moment was found to be less restricted. In the studies mentioned above, the motion of these molecules confined in all silica analogues of ZSM-5-silicalite 
was studied. Silicalite imposes strict geometrical confinements on the sorbed molecules, due to its small channel-like pores, which are, on average, $0.55 \mathrm{~nm}$ in diameter. With the kinetic diameters of the sorbed molecules being of a size that is comparable to the pore diameter in these studies, an interplay between the effects of the dipole moment and the geometrical confinement on these molecules resulted in severely restricted motion for all molecules except for propane.

$\mathrm{CO}_{2}$ is a linear molecule with no dipole moment, but with a finite quadrupole moment. This quadrupole moment of $\mathrm{CO}_{2}$ gives rise to interesting phenomena. For example, it has been suggested that due to a stronger interaction with quadrupolar $\mathrm{CO}_{2}$, this molecule replaces hydrocarbons from silica pore walls. This has been shown to give rise to an enhancement in the diffusivity of confined hydrocarbon by the addition of $\mathrm{CO}_{2}$ in several studies [8-10]. Juxtaposing the quadrupolar nature of $\mathrm{CO}_{2}$ with the effects of dipole moments on the motion of molecules in silicalite reported in [6,7], a question can be raised whether the quadrupolar nature of $\mathrm{CO}_{2}$ can also result in a severely restricted motion of $\mathrm{CO}_{2}$ in silicalite. To address this, a detailed study of $\mathrm{CO}_{2}$ dynamics in silicalite is needed. The sorption and mobility of gases such as $\mathrm{CO}_{2}$ and ethane inside ZSM- 5 has been studied by several authors [11-15]. More recently, mixtures containing $\mathrm{CO}_{2}$ in silicalite have also been studied [16-18]. Moisture-driven $\mathrm{CO}_{2}$ sorption has also been studied. For example, Shi et al. have reported the effects of moisture on the sorption of $\mathrm{CO}_{2}$ in nano-structured sorbents $[19,20]$. However, most of these studies were limited to lower loadings corresponding to pressures of a few tens of bar. Studies at higher pressures are lacking. In particular, there are very few studies addressing molecular motion under confinement at supercritical densities. The effects of high densities encountered in nanoporous engineered and natural materials are thus underrepresented in the literature.

In molecular level computer simulations, the force field used is an important parameter that can determine the course of the simulation. These force-fields are usually obtained by fitting some experimental data. For small hydrocarbons and $\mathrm{CO}_{2}$, the TraPPE (transferable potentials for phase equilibria) force field developed by Martin and Siepmann [21] is commonly used. Alkanes are generally modeled in united atom (UA) formalism in this force field, and an ethane molecule is modeled as a dumbbell-type diatomic molecule with two $\mathrm{CH}_{3}$ pseudo-atoms. An ethane molecule modeled in the TraPPE-UA force field is thus similar to the linear $\mathrm{CO}_{2}$ molecule, although with a kinetic diameter of $0.44 \mathrm{~nm}$ [22], the ethane molecule is slightly bigger than the $\mathrm{CO}_{2}$ molecule with a kinetic diameter of $0.33 \mathrm{~nm}$ [23] (the kinetic diameter is a measure of the sphere of influence of a molecule, and it represents the size of a molecule in reference to intermolecular collision events). A comparative study of $\mathrm{CO}_{2}$ and ethane can reveal the role of the quadrupole moment in the sorption and dynamics of these two molecules inside silicalite. Here, we report grand canonical Monte Carlo (GCMC) and molecular dynamics (MD) studies on the sorption, structure, and dynamics of ethane and $\mathrm{CO}_{2}$ in silicalite at higher loadings corresponding to a partial pressure of up to 100 bar. To achieve supercritical densities for both $\mathrm{CO}_{2}$ as well as ethane, this study was carried out at a temperature of $308 \mathrm{~K}$. At this temperature, the bulk state of $\mathrm{CO}_{2}$ and ethane becomes supercritical at 46 bar and 77 bar, respectively [24].

\section{Results}

\subsection{Sorption Isotherms of $\mathrm{CO}_{2}$ and Ethane in Silicalite}

Figure 1 a shows the sorption isotherms of ethane and $\mathrm{CO}_{2}$ in silicalite at $308 \mathrm{~K}$. At pressures below atmospheric pressure ( 1 bar), ethane is adsorbed in silicalite more favorably. However, at higher pressures, the sorption of $\mathrm{CO}_{2}$ is more favorable. The supercritical pressures of bulk ethane and $\mathrm{CO}_{2}$ are marked in the figure by using vertical dashed lines of black and red colors, respectively. The density of a fluid adsorbed in a nanoporous material at a given temperature and pressure condition is generally higher than the bulk density at those conditions. It is therefore customary to express the sorption isotherms in terms of the excess density $\left(n_{e x}\right)$ of the sorbed fluid over the corresponding bulk density [25]. The relation between the sorbed density $\left(n_{a d s}\right)$ and the excess density can be expressed as:

$$
n_{e x}=n_{a d s}-V_{p} \rho_{b} / M
$$


where $V_{p}$ is the volume of the pores accessible to the fluid, $M$ is its molar mass, and $\rho_{b}$ is the bulk density of the fluid at the corresponding temperature and pressure conditions. The quantity $n_{a d s}$ is obtained directly from the GCMC simulation. First et al. [26] used an automated approach to characterize several zeolitic frameworks, and obtained pore volumes. For our calculations of the excess sorption, we used their estimate of the accessible pore volume in silicalite. The values of the bulk density of several fluids including $\mathrm{CO}_{2}$ and ethane are available on the NIST web-book [24]. Using these quantities, the excess sorption isotherms of ethane and $\mathrm{CO}_{2}$ obtained at $308 \mathrm{~K}$ are shown in Figure $1 \mathrm{~b}$. Both ethane and $\mathrm{CO}_{2}$ show a region of enrichment in the pores, up to a pressure of 20 bar, followed by depletion. In what follows, we shall discuss the results from MD simulations for ethane and $\mathrm{CO}_{2}$ in silicalite, carried out on loadings corresponding to three subcritical and one supercritical pressure $(0.1,1,20$ and 100 bar). These pressures are also marked in Figure 1a.

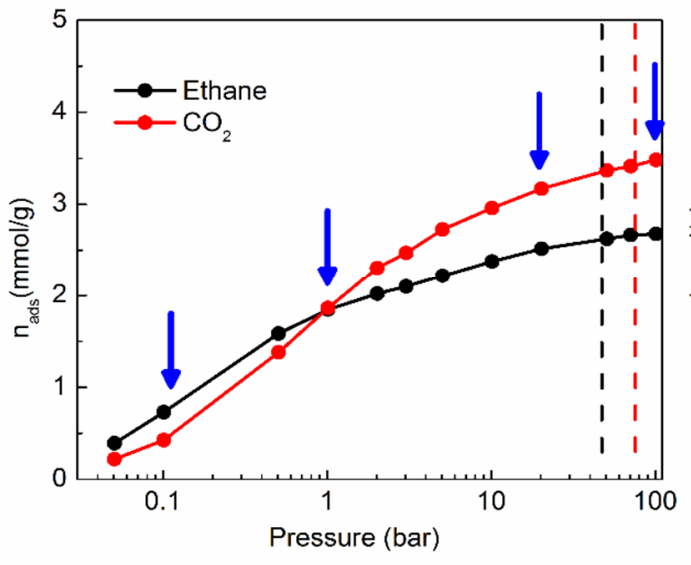

(a)

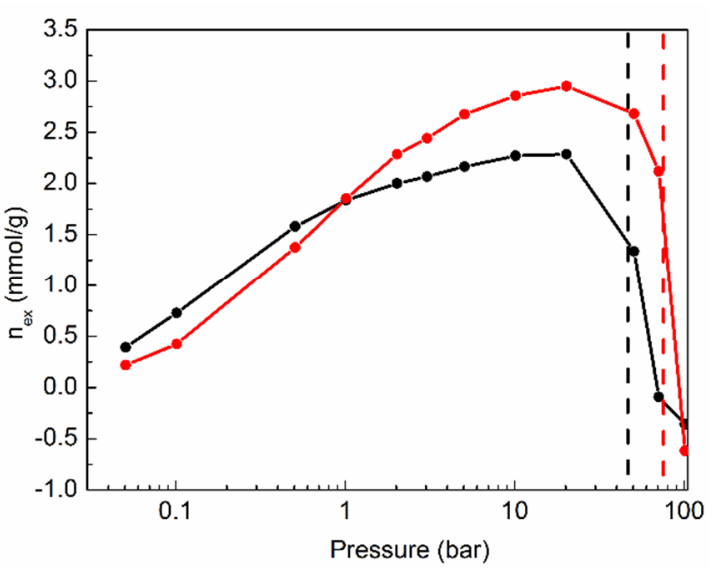

(b)

Figure 1. Sorption isotherms of ethane (black) and $\mathrm{CO}_{2}$ (red) in silicalite at $308 \mathrm{~K}$. (a) Absolute sorption; (b) Excess sorption. Symbols denote the data obtained from the GCMC simulations, while the solid lines serve as guides for the eye. The supercritical pressures of bulk ethane and $\mathrm{CO}_{2}$ are marked by black and red dashed lines, respectively, in both panels. Blue arrows indicate the gas loadings at which MD simulations were carried out for both ethane and $\mathrm{CO}_{2}$ in silicalite.

\subsection{Structure of Ethane and $\mathrm{CO}_{2}$ in Silicalite}

\subsubsection{Distribution of the Molecules in Different Pores}

Silicalite has a complex pore structure, with pores in the form of straight and sinusoidal channels, with their intersections exhibiting somewhat larger dimensions then the channels themselves. While the straight channels are oriented along the Cartesian $Y$ direction, the sinusoidal channels lie in the $X-Z$ plane. Due to the slightly different shapes of these pores, we explored which of these three pore types affords hosting of the guest molecules more readily. To probe this, we obtained the distribution of the molecules amongst these three pore types, in all simulations. These distributions are shown in Figure 2 for both ethane and $\mathrm{CO}_{2}$, with black and red symbols, respectively. At lower pressures, the difference in the number of ethane molecules is similar in all channels, within one standard deviation. However, at higher pressures, ethane molecules show a clear preference for residing in the sinusoidal channels. This could be because a molecule of large enough kinetic diameter might find it difficult to exit out of sinusoidal channels, due to its tortuosity. On the other hand, $\mathrm{CO}_{2}$ has a smaller kinetic diameter, resulting in an increased ability to move out of these channels, and therefore it shows no preference to reside in them, rather preferring intersections. 


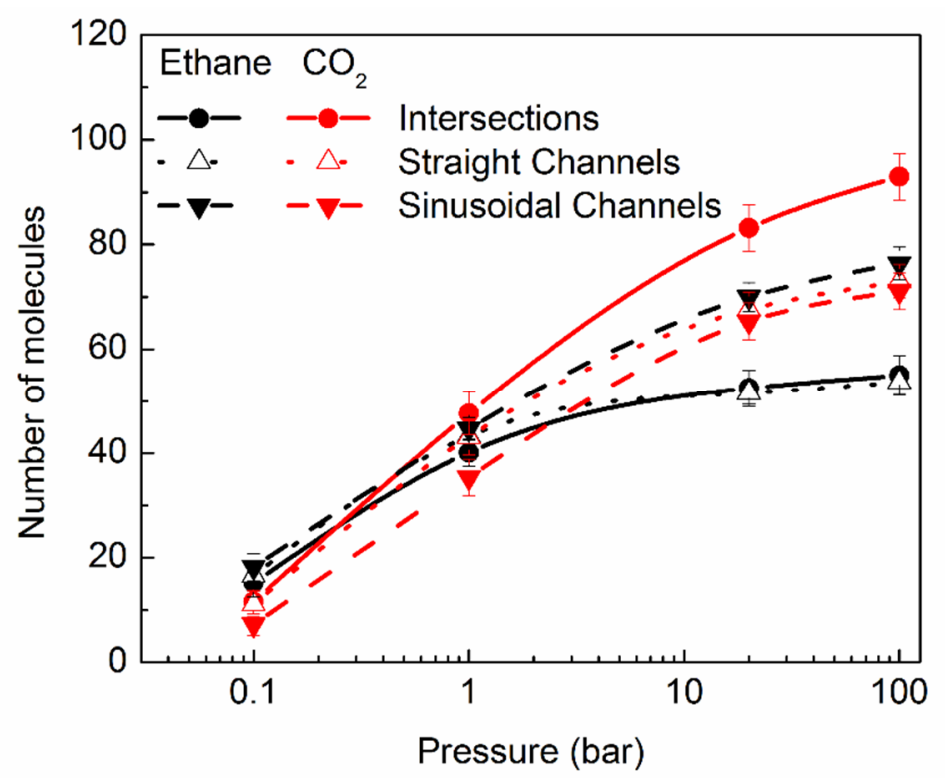

Figure 2. Distribution of the sorbed molecules among the three pore types of silicalite: intersections (circles), straight channels (open triangles), and sinusoidal channels (solid triangles). Black symbols represent the data for ethane, while red symbols show data for $\mathrm{CO}_{2}$.

\subsubsection{Orientational Distribution of Molecules in Silicalite and Anisotropy}

In a matrix like silicalite, that puts severe geometrical constraints on the sorbed molecules, both positional as well orientational ordering of molecules can be expected. Indeed, orientational ordering has been observed for several molecules that have been sorbed in silicalite $[6,15,27]$. Orientational ordering is observed overall for both ethane and $\mathrm{CO}_{2}$ in silicalite (See Supplementary Materials, Figure S1). Due to the tortuous shape of the sinusoidal channels a wide distribution of orientations can be expected for confined molecules. Likewise, at channel intersections, the geometrical restriction is slightly relaxed, due to a larger pore volume and ellipsoidal shape; thus, orientational ordering of sorbed molecules is expected to be less constrained, resulting in relatively more disorder. In straight channels that are parallel to the Cartesian $\mathrm{Y}$ axis, the ordering is relatively stronger, and the distribution is easier to interpret. Therefore, in Figure 3a, we show the orientational distribution function (ODF) of ethane and $\mathrm{CO}_{2}$ at 100 bar in the straight channels of silicalite, with respect to the three Cartesian directions. In absence of a preferred orientation, the distribution is expected to be isotropic, and the corresponding ODF is also included in Figure 3a for comparison. Any deviation from the isotropic ODF signifies a preferred orientation. Clearly, both ethane and $\mathrm{CO}_{2}$ show deviations from isotropic behavior. However, the preferred directions in the two cases are different. While ethane prefers to lie mostly along the channel axis (with the Y-direction showing stronger than isotropic distribution at lower (closer to $0^{\circ}$ ) and higher (closer to $180^{\circ}$ ) angles), $\mathrm{CO}_{2}$ prefers to lie perpendicular to the channel axis (with the Y-direction ODF peaking at $90^{\circ}$, and being weaker at lower and higher angles, compared to isotropic ODF). 


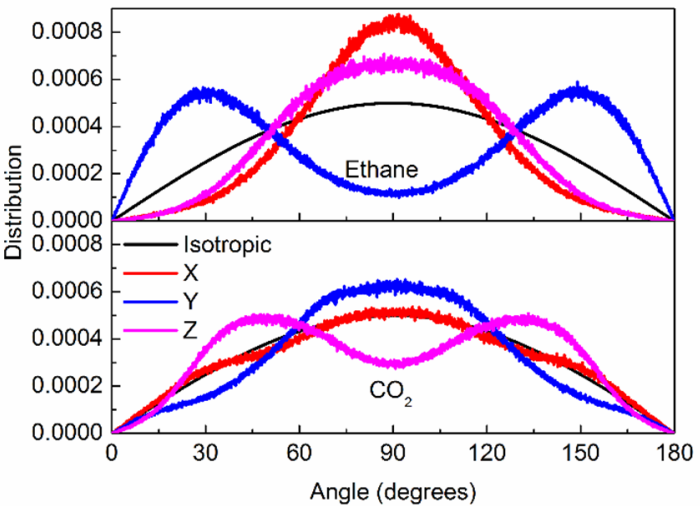

(a)

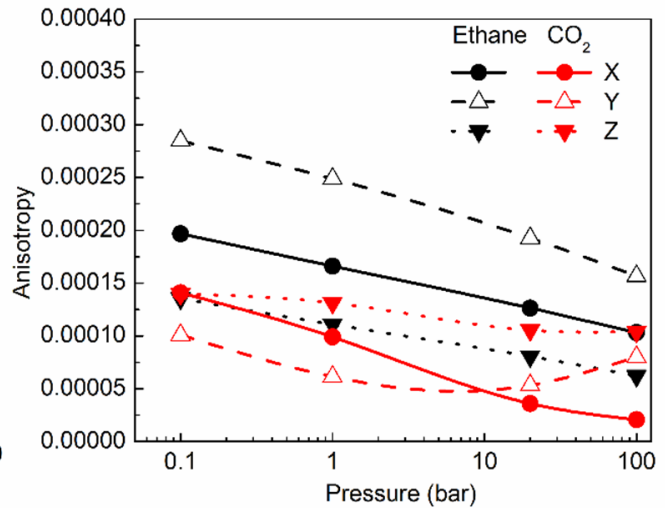

(b)

Figure 3. Orientational structure of the sorbed molecules in the straight channels of silicalite: (a) Orientational distribution with respect to the Cartesian axes X (red), Y (blue), and Z (magenta). Distribution expected in the case of an isotropic system is also included for reference as a black curve; (b) Anisotropy in the orientational distribution along different directions X (solid circles), Y (open triangles), and $\mathrm{Z}$ (solid triangles) as a function of the pressure of ethane (black) and $\mathrm{CO}_{2}$ (red). Lines in panel (b) serve as a guide to the eye.

The extent of the deviation in the orientational distribution with respect to the isotropic case can be used to quantify the anisotropy in the orientational structure of the sorbed molecules. Following previous studies $[6,15]$, we define the degree of anisotropy $(\phi)$ in the orientational structure as:

$$
\phi=\left[(1 / N) \Sigma\left(g_{i s o}(\theta)-g_{s}(\theta)\right)^{2}\right]^{1 / 2}
$$

where $g_{i}(\theta)$ is the orientational distribution function, the subscript $i$ denotes the case of isotropic distribution (iso) or the silicalite (s), and the sum is taken over $N=3150$ different values of $\theta$ over which the ODF were calculated. The degree of anisotropy that is defined in this manner is shown in Figure $3 \mathrm{~b}$ for the three Cartesian directions for ethane and $\mathrm{CO}_{2}$ in straight channels of silicalite at all pressures. For ethane, the variation of $\phi$ with pressure shows a consistent trend in all directions; namely, $\phi$ decreases with an increase in pressure. For $\mathrm{CO}_{2}$, however, the trend is different in different directions, with $\phi$ decreasing at higher pressures monotonously in the $\mathrm{Z}$ and $\mathrm{X}$ directions, while the variation in the $\mathrm{Y}$ direction is non-monotonous.

\subsection{Dynamics}

\subsubsection{Translational Motion and its Anisotropy}

Translational motion of ethane and $\mathrm{CO}_{2}$ in silicalite is studied by using the mean squared displacement $(M S D)$ of the sorbed molecules. In an anisotropic matrix such as silicalite, MSD in different directions can be expected to be different. In Figure $4 \mathrm{a}$, we show the $M S D$ of ethane and $\mathrm{CO}_{2}$ in the three Cartesian directions X, Y, and Z, along with the overall MSD at 100 bar. At this pressure, ethane can be seen to exhibit consistently higher $M S D$ than $\mathrm{CO}_{2}$ in all directions. This is in spite of the smaller kinetic diameter of $\mathrm{CO}_{2}$, as compared to ethane. A significant degree of anisotropy can be observed in the $M S D$, with the $Y$ direction showing the highest $M S D$ for both ethane and $\mathrm{CO}_{2}$, whereas the $\mathrm{Z}$ direction shows the smallest MSD. This is because of the straight channels which because of their geometry, facilitate the motion of the sorbed molecules that lie along the Cartesian $Y$ directuion, whereas the sinusoidal channels with their tortuous shape inhibiting motion, lie in the $\mathrm{X}-\mathrm{Z}$ plane. $M S D$ is a measure of the self-diffusive motion of molecules and at long times, it can be used to obtain the self-diffusion coefficient $\left(D_{s}\right)$ using the Einstein relation:

$$
D_{s}=M S D /\left(2 n_{d} t\right)
$$


where $n_{d}$ is the number of degrees of freedom, and $t$ is time. For consistency, we used the slope of $M S D$ vs. $t$ curve between $t=400 \mathrm{ps}$ and $t=600 \mathrm{ps}$, to obtain the self-diffusion coefficient in all cases. In Figure $4 b$, we show the self-diffusion coefficient of ethane and $\mathrm{CO}_{2}$ (solid black and red symbols respectively) as a function of pressure. The variation of $D_{s}$ with pressure is similar for both ethane and $\mathrm{CO}_{2} . D_{s}$ decreases at higher pressure, as can be expected, due to the crowding of molecules at higher pressures. At lower pressures, ethane exhibits slower diffusion compared to $\mathrm{CO}_{2}$, whereas at higher pressures, ethane becomes relatively faster. This could be because of the relatively lower amounts of ethane sorbed in silicalite at higher pressures. In Figure $4 \mathrm{~b}$, we also show the ratio of self-diffusivity in the directions $\mathrm{Y}$ and $\mathrm{Z}\left(D_{s}^{Y} / D_{s}^{Z}\right.$; open symbols) as a function of pressure. This ratio is a measure of the anisotropy in the translational diffusion. The anisotropy in translational diffusion is found to increase at higher pressures for both ethane and $\mathrm{CO}_{2}$. Further, the anisotropy at higher pressures is greater for ethane, compared to $\mathrm{CO}_{2}$. This could be a consequence of the slightly larger kinetic diameter of ethane, which makes it more difficult for ethane molecules to diffuse through sinusoidal channels (X-Z plane), compared to the straight channels.

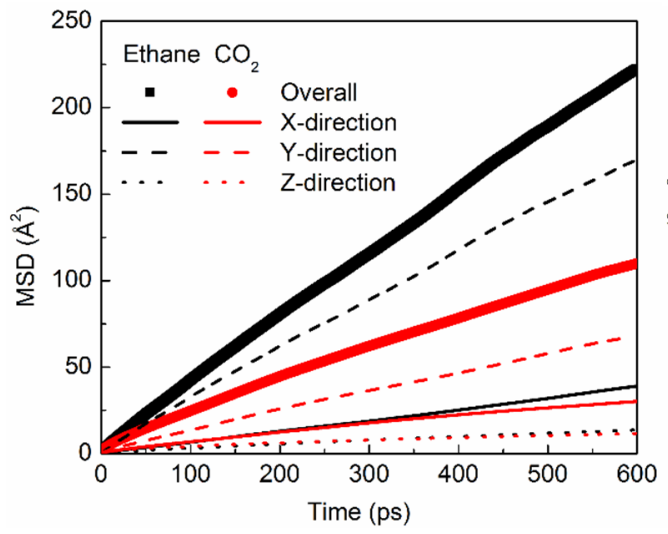

(a)

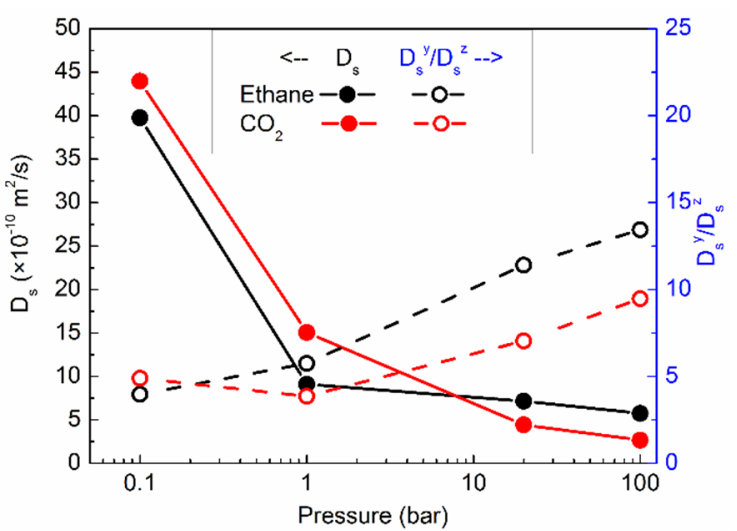

(b)

Figure 4. (a) $M S D$ of ethane (black) and $\mathrm{CO}_{2}$ (red) in silicalite in different Cartesian directions and overall. (b) The self-diffusion coefficient of ethane (solid black symbols) and $\mathrm{CO}_{2}$ (solid red symbols). Anisotropy in translational motion $\left(D_{s}{ }^{Y} / D_{s}{ }^{Z}\right)$ of ethane (open black symbols) and $\mathrm{CO}_{2}$ (open red symbols). The Y-axis labels for the self-diffusion coefficient can be read on the left, while those for anisotropy appear on the right in blue.

Another important quantity that can be used to understand the translational motion of molecules is the velocity autocorrelation function $\left(\mathrm{VACF}, \mathrm{C}_{v}(t)\right)$.

$$
C_{v}(t)=\left\langle v\left(t+t_{0}\right) \cdot v\left(t_{0}\right)>\right.
$$

where $v(t)$ is the velocity of an atom at time $t$. The angular brackets denote an average over the number of atoms, as well as the time origins $t_{0}$. The self-diffusion coefficient can also be obtained by integrating the $C_{v}(t)$ vs. $t$ curve [28]. In addition, $C_{v}(t)$ can be used to calculate the vibrational spectrum $(I(\omega))$ of the system, using the relation [29]:

$$
I(\omega)=\int C_{v}(t) \cos (\omega t) d t
$$

We point out that since both ethane and $\mathrm{CO}_{2}$ are modeled as rigid molecules here, the vibrational spectra obtained using the above equation will be limited to the intermolecular vibrational modes alone. In Figure 5, we show the vibrational spectra calculated from the VACF for ethane (top panel) and $\mathrm{CO}_{2}$ (bottom panel) at different pressures. While the spectra for ethane are bimodal at low pressures, the lower energy peak merges with the broad peak at higher pressures. $\mathrm{CO}_{2}$ on the other hand exhibits a single broad peak at all pressures. Further, a shift to higher energies can be observed in the spectra for both ethane and $\mathrm{CO}_{2}$ at higher pressures. A similar shift to higher energies has been observed in the 
vibrational spectrum of propane confined in MCM-41-S in an inelastic neutron scattering experimental study [29]. For comparison, the vibrational spectra of bulk ethane [30] and $\mathrm{CO}_{2}$ [31] obtained from inelastic neutron scattering experiments are also included. These spectra are taken from the ISIS INS database [32]. Although the spectra calculated from a simulation of rigid molecules is not expected to capture all the details of the experimental spectra, some salient features are reproduced. For example, the intermolecular vibrational modes of $\mathrm{CO}_{2}$ confined in silicalite peak at lower energies compared to ethane, a result that is qualitatively consistent with the experimental data on bulk ethane and $\mathrm{CO}_{2}$. Further, the calculated spectra of both ethane and $\mathrm{CO}_{2}$ look more disordered, as compared to the bulk spectra. A similar confinement-induced disorder was observed in the vibrational spectra of propane in MCM-41-S [29] obtained from INS experiments, as well as MD simulations.

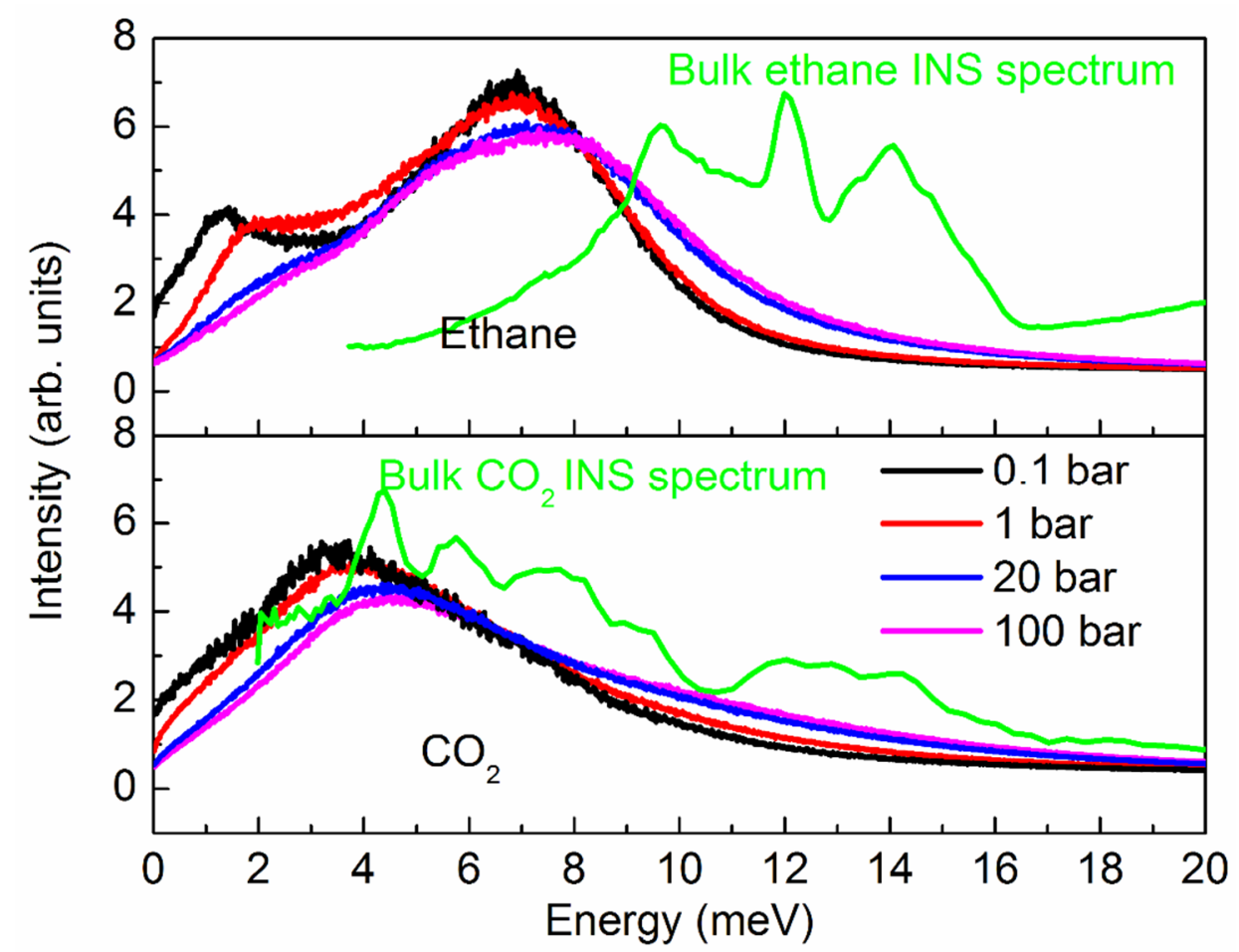

Figure 5. Vibrational spectra of ethane (top) and $\mathrm{CO}_{2}$ (bottom) in silicalite at different pressures. Also included in green are the spectra of bulk ethane [30] and $\mathrm{CO}_{2}$ [31], obtained in inelastic neutron scattering experiments and available at the ISIS INS database [32].

\subsubsection{Rotational Motion}

The preferred orientations seen in the ODF in Figure 2 for both ethane and $\mathrm{CO}_{2}$ suggest that the rotational motion of the sorbed molecules is restricted. The rotational motion of rigid molecules in general can be studied by following the orientation of a unit vector $(\boldsymbol{u})$ that is attached to the molecule as a function of time $[33,34]$. In particular, one can follow the evolution of the first-order rotational correlation function $\left(\mathrm{RCF}, \mathrm{C}_{R}(t)\right)$, defined as:

$$
C_{R}(t)=<u\left(t+t_{0}\right) \cdot u\left(t_{0}\right)>
$$

In Figure 6a we show the RCF of ethane (black curve) and $\mathrm{CO}_{2}$ (red curve) at 100 bar. Compared to ethane, the RCF of $\mathrm{CO}_{2}$ takes longer to decay to zero, indicating a slower rotational motion of $\mathrm{CO}_{2}$ compared to ethane. Further, the RCF of both ethane and $\mathrm{CO}_{2}$ exhibit distinct behaviors at times that are shorter than a picosecond, and longer times. This facilitates dividing the RCF in two regimes of sub-picosecond times, and longer times. The boundary of these two regimes is marked clearly in case of ethane in the form of a kink. As mentioned in previous publications, this is a signature of vibrational 
motion $[15,27]$. No kink can be observed in the case of $\mathrm{CO}_{2}$ suggesting that the rotational motion of $\mathrm{CO}_{2}$ is different from the libration type of motion exhibited by ethane and other molecules $[6,15,27]$. $\mathrm{CO}_{2}$ has a kinetic diameter that is considerably smaller than the channel diameter of silicalite. Further, the interaction of the molecule with the pore walls is not strong enough to restrict it from rotating more freely inside the silicalite channels, tracing out the entire orientational space available.

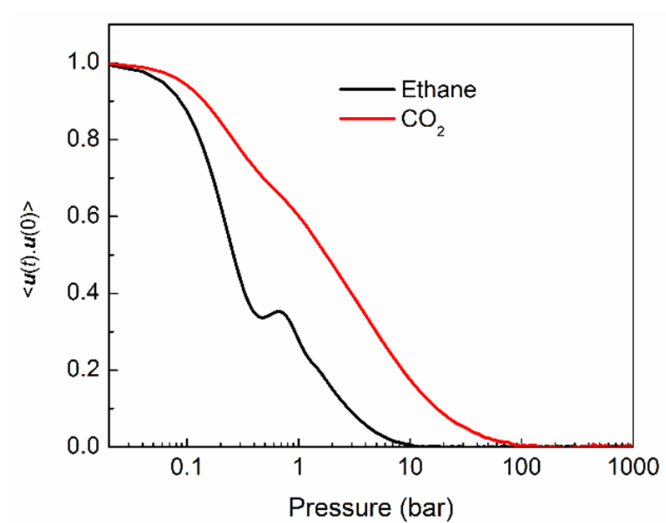

(a)

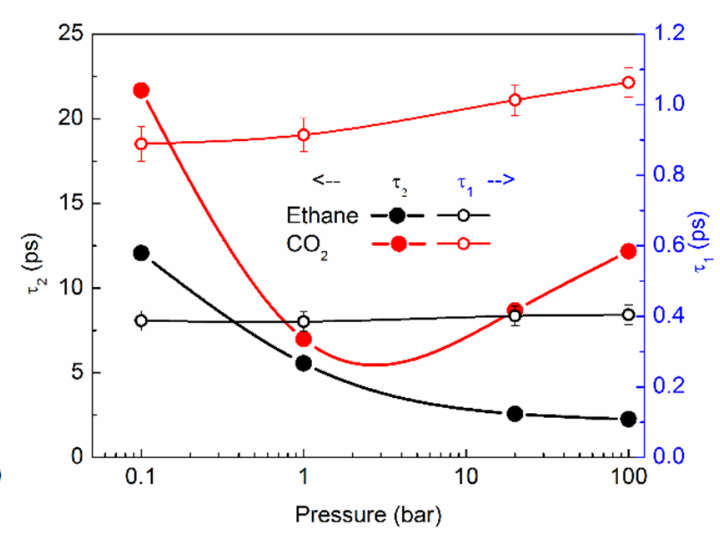

(b)

Figure 6. Rotational motion of ethane (black) and $\mathrm{CO}_{2}$ (red) in silicalite: (a) Rotational correlation function (RCF) at 100 bar. (b) Time scales of rotational motion obtained by fitting the two regimes of RCF with an exponential decay. In the first regime, $\tau_{1}$ was obtained by fitting the RCF between 0 and 0.2 ps. The Y-axis labels for $\tau_{1}$ can be read on the right in blue, while those for $\tau_{2}$ appear on the left.

To estimate the time scales of rotational motion, we fitted the RCF in the two regimes with exponential decay functions. The time scales in the two regimes of rotational motion, $\tau_{1}$ (shorter time scale regime, open symbols) and $\tau_{2}$ (longer time regime, solid symbols) are shown in Figure $6 \mathrm{~b}$ for ethane (black) and $\mathrm{CO}_{2}$ (red) at different pressures. For consistency, all RCFs were fit between $t=0$ and $t=0.2 \mathrm{ps}$; and $t=2 \mathrm{ps}$ and $t=200 \mathrm{ps}$ to obtain $\tau_{1}$ and $\tau_{2}$, respectively. The rotational motion of $\mathrm{CO}_{2}$ is slower than that of ethane in both regimes. Although the short time rotational motion of $\mathrm{CO}_{2}$ shows a monotonous decrease at higher pressures, the longer time rotation becomes faster initially, when pressure is increased to 1 bar, and for higher pressures, it slows down again. For ethane, the short time rotational motion is independent of pressure, while the longer time rotation becomes faster consistently with an increase in pressure.

\section{Discussion}

The sorption isotherms presented in Figure 1 are in very good agreement with those reported by Sun et al. [13] for ethane in silicalite at $308 \mathrm{~K}$ up to $3.43 \mathrm{bar}$, and $\mathrm{CO}_{2}$ in silicalite up to 1.37 bar. Ethane shows a stronger sorption at lower pressures, while $\mathrm{CO}_{2}$ is adsorbed more readily at higher pressures. In a study on $\mathrm{CO}_{2}$ sorption in metal organic frameworks at $298 \mathrm{~K}$ up to 35 bar, Walton et al. [35] found that a higher amount of $\mathrm{CO}_{2}$ is adsorbed in the metal organic framework, if electrostatic interactions are accounted for in the simulation. Conversely, lower amounts of $\mathrm{CO}_{2}$ were found to be adsorbed if the electrostatic interactions were switched off. The higher sorption of $\mathrm{CO}_{2}$ is thus due to the electrostatic interactions between the quadrupole moment of $\mathrm{CO}_{2}$ and the pore surface.

Both ethane and $\mathrm{CO}_{2}$ exhibit type I isotherms up to 100 bar. It is interesting to note that all three types of pores in silicalite intersections, straight channels, and sinusoidal channels are accessible to both ethane, as well as $\mathrm{CO}_{2}$ at all pressures, as shown in Figure 2. Even at lower pressures not investigated with MD, GCMC data show that the molecules start to populate all three types of pores (see Supplementary Materials, Figure S2). This is in contrast to the case of branched alkanes, e.g., iso-butane, that populate only the intersections at lower pressures, resulting in a type IV isotherm, as observed by Vlugt et al. [36]. This is because of the relatively smaller kinetic diameter of ethane and 
$\mathrm{CO}_{2}$, compared to iso-butane. It is natural that $\mathrm{CO}_{2}$ shows a clear preference for intersections at higher pressure. This is because the intersections offer a marginally larger ellipsoidal free space, compared to the straight and sinusoidal channels. However, ethane molecules at higher pressures prefer to stay in sinusoidal channels, although at lower pressures, this preference is not very clear. We note that in our previous study of ethane in silicalite, the highest loading investigated (eight molecules per unit cell) corresponded to a pressure of slightly less than 1 bar. At that pressure, the error bars in Figure 2 make no conclusion about the preference for pore type difficult.

The orientational order exhibited by both ethane and $\mathrm{CO}_{2}$ (Figure 3) is consistent with that exhibited by several other molecules confined in silicalite $[6,15,27]$. This behavior results from a strict geometrical restriction imposed by the narrow silicalite pores. Further, the preferred orientation of $\mathrm{CO}_{2}$ in straight channels, perpendicular to the channel direction, is a consequence of the partial charges on the extremities of the molecule. The electrostatic interactions between the extremities of $\mathrm{CO}_{2}$ molecule and the pore wall make the molecule lie perpendicular to the channel, whereas in the absence of such partial charges, ethane molecules prefer a parallel orientation. This has important consequences for the motion of these molecules. Being linear molecules, it can be expected that the motions of both these molecules along their molecular axes will be easier. Indeed, for ethane, it was found that the MSD parallel to the molecular axis is considerably higher than that which is perpendicular to the axis (see Supplementary Materials, Figure S3). However, for $\mathrm{CO}_{2}$, no considerable difference was observed for MSD that was parallel or perpendicular to the molecular axis. Although, in a free environment, the motion of a $\mathrm{CO}_{2}$ molecule along the molecular axis would be easier, in case of silicalite, no free space is available in that direction, as the molecule lies perpendicular to the channel axis.

At lower pressures, $\mathrm{CO}_{2}$ diffuses faster than ethane. However, at those pressures, the amount of $\mathrm{CO}_{2}$ adsorbed in silicalite is lower than that of ethane. Thus, crowding effects on the translation diffusion for ethane are stronger. However, at higher pressures, the relative amount of $\mathrm{CO}_{2}$ is larger than ethane, and hence the diffusivity of $\mathrm{CO}_{2}$ is slower. The variation of self-diffusivity can be viewed as the interplay of the kinetic diameters and crowding effects. The rotational motion of $\mathrm{CO}_{2}$ in silicalite is slower than ethane, in spite of a slightly smaller kinetic diameter. While for ethane, the rotational motion gets consistently faster at higher pressures, for $\mathrm{CO}_{2}$ the variation is non-monotonous. In a previous work on ethane rotation in silicalite [15], we observed a correlation between the variation of rotational motion and anisotropy in the ODF. We note the same correlation here in the case of ethane, wherein as the anisotropy decreases at higher pressure, the rotational motion becomes faster. A similar correlation can also be seen between the rotational motion of $\mathrm{CO}_{2}$ and anisotropy in ODF in the $\mathrm{Y}$ direction.

Although the behavior of both ethane and $\mathrm{CO}_{2}$ in silicalite at pressures corresponding to bulk supercritical pressures is quantitatively different from that at lower pressures, no major qualitative changes were observed at this pressure (100 bar) compared to the lower pressures corresponding to bulk sub-critical pressures.

In the absence of electrostatic interactions, $\mathrm{CO}_{2}$, which is a smaller molecule, can be expected to experience relatively less restriction to motion by the confining media. However, due to the quadrupole moment of $\mathrm{CO}_{2}$ that appears as partial charges on the $\mathrm{C}$ and $\mathrm{O}$ atoms in the TraPPE model used here, $\mathrm{CO}_{2}$ is not only adsorbed in silicalite to a greater extent, its motion is also suppressed more, compared to ethane. On the other hand, the suppression in the mobility of $\mathrm{CO}_{2}$ is not as drastic as what is observed in the case of molecules with dipole moments that barely move inside silicalite [6,7]. Thus, although not as strongly as the dipole moment of other molecule, the quadrupole moment of $\mathrm{CO}_{2}$ does affect its sorption, structure, and dynamics in silicalite.

\section{Materials and Methods}

In this work we used Grand Canonical Monte Carlo (GCMC) simulations to obtain the sorption isotherms of ethane and $\mathrm{CO}_{2}$ in silicalite, and Molecular Dynamics (MD) simulations to study their structural and dynamical properties. While GCMC simulations were carried out using DL_Monte [37], 
MD simulations were carried out using DL_Poly [38]. Both these packages have been developed by CCP Forge, and they are available free of charge. DL_Monte has been shown to have a computational performance that is comparable to several other Monte Carlo codes [39]. Besides, having commonalities with DL_Poly, it was a natural choice to complement the MD simulations carried out using DL_Poly.

A model ZSM-5 pore network was built by making use of the co-ordinates provided by Koningsveld et al. [40]. To start with, two molecules each of $\mathrm{CO}_{2}$ or ethane were loaded in the straight channels of ZSM-5. GCMC simulations were then carried out on the simulation cell thus obtained. During the simulation, the sorbed molecules, i.e., ethane or $\mathrm{CO}_{2}$, could be inserted, deleted, translated, or rotated, while all silicalite atoms were kept rigid. All simulations were carried out using a fixed gas partial pressure at $308 \mathrm{~K}$. This is done by using the following selection procedures for the insertion $\left(P_{i}\right)$ or deletion $\left(P_{d}\right)$ of a molecule.

$$
\begin{gathered}
P_{i}=\min \{1,(\beta V P) \exp (-\beta \Delta U) /(N+1)\} \\
P_{d}=\min \{1,(N / \beta V P) \exp (-\beta \Delta U)\}
\end{gathered}
$$

where $V$ is the simulation cell volume, $P$ is the partial pressure of the gas, $U$ represents the potential energy, $N$ is the number of molecules and $\beta=k_{B} T$.

To start with, GCMC simulations were carried out at the lowest pressure of 0.05 bar. After this, successive simulations were carried out by sequentially increasing the pressure. The final configurations of each simulation were used as the initial configurations for the next simulation. At lower pressures, 2 million Monte Carlo steps were enough to obtain statistically meaningful configurations. Of these, the first 500,000 steps were discarded to account for equilibrium. At higher pressures, 5 million steps were used, out of which, the first 150,000 were equilibration steps. Coordinates were sampled every 10,000 steps. During the simulation, the translation, rotation, insertion, and deletion of ethane or $\mathrm{CO}_{2}$ were undertaken with a probability of $0.25,0.25,0.5$, and 0.5 respectively. A potential cut-off of $1.4 \mathrm{~nm}$, as suggested for TraPPE-UA force field was used. The number of molecules of ethane or $\mathrm{CO}_{2}$ in the simulation cell averaged over the entire production run, discarding the equilibration steps, were used to obtain the adsorption isotherms as a function of pressure.

Having obtained the adsorption isotherms, four pressures of interest $(0.1,1,20$, and 100 bar) were selected to further investigate the structures and dynamics of ethane and $\mathrm{CO}_{2}$ in silicalite, using $\mathrm{MD}$ simulations. The outputs from the MC simulations were used as the inputs for the MD simulations in all cases. MD simulations were carried out in NVT ensemble, using a Nose-Hoover thermostat. A total of eight simulations, four simulations corresponding to the selected pressure each for ethane and $\mathrm{CO}_{2}$ in silicalite, were carried out by using a simulation time step of $1 \mathrm{fs}$. In each simulation, the system was allowed to equilibrate at $308 \mathrm{~K}$ for $0.5 \mathrm{~ns}$. After this, trajectories were recorded at each $0.02 \mathrm{ps}$ for a duration of $1.5 \mathrm{~ns}$. Various properties of interest were then calculated from these trajectories. The achievement of equilibrium was confirmed when the energy and temperature values showed no clear monotonous increase/decrease in time, and the variations were less than $5 \%$.

\section{Conclusions}

We studied the sorption, structure, and dynamics of ethane and $\mathrm{CO}_{2}$ in silicalite-two similar molecules without and with a quadrupole moment. The quadrupole moment of $\mathrm{CO}_{2}$ plays an important role in all of the properties investigated here. Combining the information obtained here with some previous studies, we can conclude that the effects of electrostatic interactions on the properties of a confined fluid in a nanoporous medium are strongest for molecules with a net dipole moment, and become progressively milder for molecules with a quadrupole moment and no polarity.

Supplementary Materials: The Supplementary Materials are available online. Figure S1: Orientational distribution functions of ethane and $\mathrm{CO}_{2}$ in all pores of silicalite, Figure S2: Distribution of ethane and $\mathrm{CO}_{2}$ in 
straight and sinusoidal channels, and their intersections at 0.05 bar; Figure S3: $M S D$ of ethane and $\mathrm{CO}_{2}$ parallel and perpendicular to the molecular axis at 100 bar.

Author Contributions: Conceptualization, S.G.; Formal analysis, S.G.; Funding acquisition, D.C.; Investigation, S.G.; Methodology, S.G.; Software, S.G.; Validation, S.G.; Writing—original draft, Siddharth Gautam; Writing-review \& editing, S.G., T.L. and D.C.

Funding: This research was funded by the U.S. Department of Basic Energy, Office of Science, Office of Basic Energy Sciences, Division of Chemical Sciences, Geosciences and Biosciences, Geosciences Program, grant number DESC000687, and A. P. Sloan Foundation funded Deep Carbon Observatory.

Acknowledgments: We would like to acknowledge STFC's Daresbury Laboratory for providing the packages DL-Monte and DL-Poly, which were used in this work. We would also like to acknowledge computational support from the Deep Carbon Observatory cluster, hosted by Rensselaer Polytechnic Institute (Peter Fox and Patrick West).

Conflicts of Interest: The authors declare no conflict of interest. The funders had no role in the design of the study; in the collection, analyses, or interpretation of data; in the writing of the manuscript, or in the decision to publish the results.

\section{References}

1. Gautam, S.S.; Ok, S.; Cole, D.R. Structure and Dynamics of Confined C-O-H Fluids Relevant to the Subsurface: Application of Magnetic Resonance, Neutron Scattering, and Molecular Dynamics Simulations. Front. Earth Sci. 2017, 5, 1-19. [CrossRef]

2. Kolesnikov, A.I.; Zanotti, J.-M.; Loong, C.-K.; Thiyagarajan, P.; Moravsky, A.P.; Loutfy, R.O.; Burnham, C.J. Anomalously soft dynamics of Water in a Nanotube: A Revelation of Nanoscale Confinement. Phys. Rev. Lett. 2004, 93, 035503. [CrossRef] [PubMed]

3. Granick, S. Motions and Relaxations of Confined Liquids. Science 1991, 253, 1374-1379. [CrossRef]

4. Pitakbunkate, T.; Balbuena, P.B.; Moridis, G.J.; Blasingame, T.A. Effect of Confinement on Pressure/Volume/Temperature Properties of Hydrocarbons in Shale Reservoirs. SPE J. 2016, 21, 621-634. [CrossRef]

5. Cole, D.R.; Mamontov, E.; Rother, G. Structure and dynamics of fluids in microporous and mesoporous earth and engineered materials. In Neutron Applications in Earth, Energy and Environmental Sciences; Liang, L., Rinaldi, R., Schober, H., Eds.; Springer: Boston, MA, USA, 2009; pp. 547-570, ISBN 978-0-387-09416-8.

6. Dhiman, I.; Bhowmik, D.; Shrestha, U.R.; Cole, D.R.; Gautam, S. Effect of molecular shape on rotation under severe confinement. Chem. Eng. Sci. 2018, 180, 33-41. [CrossRef]

7. Dhiman, I.; Shrestha, U.R.; Bhowmik, D.; Cole, D.R.; Gautam, S. Influence of molecular shape on self-diffusion under severe confinement: A molecular dynamics study. Chem. Phys. 2019, 516, 92-102. [CrossRef]

8. Le, T.; Striolo, A.; Cole, D.R. $\mathrm{CO}_{2}-\mathrm{C}_{4} \mathrm{H}_{10}$ Mixtures Simulated in Silica Slit Pores: Relation between Structure and Dynamics. J. Phys. Chem. C 2015, 119, 15274-15284. [CrossRef]

9. Gautam, S.; Liu, T.; Rother, G.; Jalarvo, N.; Mamontov, E.; Welch, S.; Sheets, J.; Droege, M.; Cole, D.R. Dynamics of Propane in Nanoporous Silica Aerogel: A Quasielastic Neutron Scattering Study. J. Phys. Chem. C 2015, 119, 18188-18195. [CrossRef]

10. Patankar, S.; Gautam, S.; Rother, G.; Podlesnyak, A.; Ehlers, G.; Liu, T.; Cole, D.R.; Tomasko, D.L. Role of Confinement on Adsorption and Dynamics of Ethane and an Ethane- $\mathrm{CO}_{2}$ Mixture in Mesoporous CPG Silica. J. Phys. Chem. C 2016, 120, 4843-4853. [CrossRef]

11. Choudhary, V.R.; Mayadevi, S. Adsorption of methane, ethane, ethylene, and carbon dioxide on silicalite-I. Zeolites 1996, 17, 501-507. [CrossRef]

12. Hirotani, A.; Mizukami, K.; Miura, R.; Takaba, H.; Miya, T.; Fahmi, A.; Stirling, A.; Kubo, M.; Miyamoto, A. Grand canonical Monte Carlo simulation of the adsorption of $\mathrm{CO}_{2}$ on silicalite and NaZSM-5. Appl. Surf. Sci. 1997, 120, 81-84. [CrossRef]

13. Sun, M.S.; Shah, D.B.; Xu, H.H.; Talu, O. Adsorption Equilibria of $\mathrm{C} 1$ to $\mathrm{C} 4$ Alkanes, $\mathrm{CO}_{2}$, and $\mathrm{SF}_{6}$ on Silicalite. J. Phys. Chem. B 1998, 102, 1466-1473. [CrossRef]

14. Pascual, P.; Ungerer, P.; Tavitian, B.; Pernot, P.; Boutin, A. Development of a transferable guest-host force field for adsorption of hydrocarbons in zeolites: I. Reinvestigation of alkane adsorption in silicalite by grand canonical Monte Carlo simulation. Phys. Chem. Chem. Phys. 2003, 5, 3684-3693. [CrossRef] 
15. Gautam, S.; Liu, T.; Patankar, S.; Tomasko, D.; Cole, D. Location dependent orientational structure and dynamics of ethane in ZSM5. Chem. Phys. Lett. 2016, 648, 130-136. [CrossRef]

16. García-Pérez, E.; Parra, J.B.; Ania, C.O.; García-Sánchez, A.; van Baten, J.M.; Krishna, R.; Dubbeldam, D.; Calero, S. A computational study of $\mathrm{CO}_{2}, \mathrm{~N}_{2}$, and $\mathrm{CH}_{4}$ adsorption in zeolites. Adsorption 2007, 13, 469-476. [CrossRef]

17. Selassie, D.; Davis, D.; Dahlin, J.; Feise, E.; Haman, G.; Sholl, D.S.; Kohen, D. Atomistic Simulations of $\mathrm{CO}_{2}$ and $\mathrm{N}_{2}$ Diffusion in Silica Zeolites: The Impact of Pore Size and Shape. J. Phys. Chem. C 2008, 112, 16521-16531. [CrossRef]

18. Razavian, M.; Fatemi, S.; Masoudi-Nejad, M. A Comparative Study of $\mathrm{CO}_{2}$ and $\mathrm{CH}_{4}$ Adsorption on Silicalite-1 Fabricated by Sonication and Conventional Method. Adsorpt. Sci. Technol. 2014, 32, 73-87. [CrossRef]

19. Shi, X.; Xiao, H.; Liao, X.; Armstrong, M.; Chen, X.; Lackner, K.S. Humidity Effect on Ion Behaviors of Moisture-driven $\mathrm{CO}_{2}$ Sorbents. J. Chem. Phys. 2018, 149, 164708. [CrossRef]

20. Shi, X.; Xiao, H.; Lackner, K.S.; Chen, X. Capture $\mathrm{CO}_{2}$ from Ambient Air Using Nanoconfined Ion Hydration. Angew. Chem. 2016, 128, 4094-4097. [CrossRef]

21. Martin, M.G.; Siepmann, J.I. Transferable Potentials for Phase Equilibria. 1. United-Atom Description of n-Alkanes. J. Phys. Chem. B 1998, 102, 2569-2577. [CrossRef]

22. Aguado, S.; Bergeret, G.; Daniel, C.; Farrusseng, D. Absolute Molecular Sieve Separation of Ethylene/Ethane Mixtures with Silver Zeolite A. J. Am. Chem. Soc. 2012, 134, 14635-14637. [CrossRef] [PubMed]

23. Yang, J.; Zhao, Q.; Xu, H.; Li, L.; Dong, J.; Li, J. Adsorption of $\mathrm{CO}_{2}, \mathrm{CH}_{4}$, and $\mathrm{N}_{2}$ on Gas Diameter Grade Ion-exchange Small Pore Zeolites. J. Chem. Eng. Data 2012, 57, 3701-3709. [CrossRef]

24. Lemmon, E.W.; McLinden, M.O.; Friend, D.G. Thermophysical Properties of Fluid Systems in NIST Chemistry WebBook, NIST Standard Reference Database Number 69; Linstrom, P.J., Mallard, W.G., Eds.; National Institute of Standards and Technology: Gaithersburg, MD, USA, 2017.

25. Pini, R. Interpretation of net and excess adsorption isotherms in microporous adsorbents. Microporous Mesoporous Mater. 2014, 187, 40-52. [CrossRef]

26. First, E.L.; Gounaris, C.E.; Wei, J.; Floudas, C.A. Computational characterization of zeolite porous networks: An automated approach. Phys. Chem. Chem. Phys. 2011, 13, 17339-17358. [CrossRef] [PubMed]

27. Gautam, S.; Sharma, V.K.; Mitra, S.; Chaplot, S.L.; Mukhopadhyay, R. Rotational dynamics of propylene in ZSM-5 zeolitic frameworks. Chem. Phys. Lett. 2011, 501, 345-350. [CrossRef]

28. Gautam, S.; Mitra, S.; Chaplot, S.L.; Mukhopadhyay, R. Dynamics of 1,3-butadiene adsorbed in Na-Y zeolite: A molecular dynamics simulation study. Phys. Rev. E 2008, 77, 061201. [CrossRef] [PubMed]

29. Gautam, S.; Kolesnikov, A.I.; Rother, G.; Dai, S.; Qiao, Z.A.; Cole, D. Effects of Confinement and Pressure on the Vibrational Behavior of Nano-Confined Propane. J. Phys. Chem. A 2018, 122, 6736-6745. [CrossRef]

30. Mitchell, P.C.H.; Parker, S.F.; Ramirez-Cuesta, A.J.; Tomkinson, J. Vibrational Spectroscopy with Neutrons: With Applications in Chemistry, Biology, Materials Science and Catalysis; World Scientific: Singapore, 2005; Volume 3, ISBN 9812567836.

31. Tomkinson, J.; ISIS, Chilton, UK. Unpublished work.

32. ISIS INS Database. Available online: https://www.isis.stfc.ac.uk/Pages/INS-database.aspx (accessed on 29 November 2018).

33. Gautam, S.; Cole, D. Molecular dynamics simulation study of meso-confined propane in $\mathrm{TiO}_{2}$. Chem. Phys. 2015, 458, 68-76. [CrossRef]

34. Gautam, S.; Le, T.; Striolo, A.; Cole, D. Molecular dynamics simulations of propane in slit shaped silica nano-pores: Direct comparison with quasielastic neutron scattering experiments. Phys. Chem. Chem. Phys. 2017, 19, 32320-32332. [CrossRef]

35. Walton, K.S.; Millward, A.R.; Dubbeldam, D.; Frost, H.; Low, J.J.; Yaghi, O.M.; Snurr, R.Q. Understanding Inflections and Steps in Carbon Dioxide Adsorption Isotherms in Metal-Organic Frameworks. J. Am. Chem. Soc. 2008, 130, 406-407. [CrossRef]

36. Vlugt, T.J.H.; Zhu, W.; Kapteijn, F.; Moulijn, J.A.; Smit, B.; Krishna, R. Adsorption of Linear and Branched Alkanes in the Zeolite Silicalite-1. J. Am. Chem. Soc. 1998, 120, 5599-5600. [CrossRef]

37. Purton, J.A.; Crabtree, J.C.; Parker, S.C. DL_Monte: A general purpose program for parallel Monte Carlo simulation. Mol. Simul. 2013, 39, 1240-1252. [CrossRef]

38. Todorov, I.T.; Smith, W.; Trachenko, K.; Dove, M.T. DL_POLY_3: New dimensions in molecular dynamics simulations via massive parallelism. J. Mater. Chem. 2006, 16, 1911-1918. [CrossRef] 
39. Gowers, R.J.; Farmahini, A.H.; Friedrich, D.; Sarkisov, L. Automated analysis and benchmarking of GCMC simulation programs in application to gas adsorption. Mol. Simul. 2018, 44, 309-321. [CrossRef]

40. Van Koningsveld, H.; van Bekkum, H.; Jansen, J.C. On the Location and Disorder of the Tetrapropylammonium (TPA) Ion in zeolite ZSM-5 with Improved Framework Accuracy. Acta Crystallogr. Sect. B 1987, B43, 127-132. [CrossRef] 